\title{
Measuring the Phase Transformation Kinetics Under Non-Equilibrium Conditions from Time Resolved High Resolution TEM Images
}

\author{
Renu Sharma ${ }^{1}$, Zahra Hussani ${ }^{1}$, Pin Ann Lin $^{1.2}$ and Bharath Natarajan ${ }^{1,2}$ \\ 1. Center for Nanoscale Science and Technology, National Institute of Standards and Technology, \\ Gaithersburg, MD 20899-6203. \\ 2. Maryland NanoCenter, University of Maryland, College Park, MD 20742, USA
}

The interaction of gases with a solid catalyst nanoparticle during catalysis is a non-equilibrium system that requires high spatial and temporal resolution measurements to elucidate underlying mechanisms. State-of-the-art environmental transmission electron microscopy (ETEM) enables in situ measurements of the dynamic changes occurring under reaction conditions [1,2]. These changes usually take place rapidly at the nanometer scale. In order to record them in real time, both the high image resolution and frame rates are needed. Recently, direct electron detection cameras, have enabled us to record atomic resolution images with ms time resolution but result in producing large video data sets (gigabytes per second). To follow the atomic-level changes occurring under reaction conditions over time, the structure and phase of the nanostructure under observation must be analyzed for each individual frame. It is laborious to analyze such large videos frame by frame. An automated image processing scheme (AIPS) is therefore desirable to increase the speed and reliability of the analysis. There are two major inherent problems for such an automated structural analysis: (a) individual frames are noisy due to the short frame acquisition time and (b) the sample drifts during video recording periods that can range from seconds to minutes. We have developed an automatic method to mitigate these problems using a combination of publicly available and NIST-developed algorithms to obtain structural information from the images extracted from videos. Here we present the kinetics of phase transformation in catalyst nanoparticles under non-equilibrium reactive environment.

We use an ETEM to record atomic resolution videos of catalyst nano particles under reaction conditions. The images are analyzed using fast Fourier transform (FFT) and image simulation to identify and confirm the structures of different phases present. Individual frames extracted from the videos are filtered and drift corrected, using publically available programs such as ImageJ [3], to obtain a stack of images for automated image processing scheme (AIPS). Atomic coordinates are obtained using template matching followed calculating centroid of each atomic column. Structure of phases present was then identified from the nearest neighbor distances to measure the reaction kinetics.

Carbide phase has been reported to form during the nucleation and growth of carbon nanotubes [4,5]. Here we show that the formation and decomposition of metal carbide is an intermediate step for the formation carbon-carbon bonds, i.e. nucleation of single wall carbon nanotube (SWCNT). Figure 1a shows an atomic resolution image of a catalyst nanoparticle during a SWCNT growth at $650{ }^{\circ} \mathrm{C}$ in 0.01 $\mathrm{Pa} \mathrm{C}_{2} \mathrm{H}_{2}$ gas pressure. Measured inter-atomic distances, i.e. nearest neighbor distances (Figure 1b) were used to identify the areas occupied by $\mathrm{Co}$ and $\mathrm{CoC}_{2}$ at any given time (Figure 1c). Figure 2 (left) shows a series of images extracted from the videos at different time showing the fluctuation in the two regions with time. We find that fluctuations in the measured carbon content (Figure 2, right) for either of the reactions is aperiodic and in anti-sync, i.e. SWCNT growth is negligible as the carbide region in the particle increase. On the other hand, the tube grows as the carbide region decrease. Such precise $(0.07$ $\mathrm{pm}$ ) measurement of interatomic distances can be used not only to measure phase transformation rates in 
a catalyst nanoparticle during the growth of SWCNT but also for other reactions such as reduction of iron oxide nanowires. Videos recorded using two different cameras have been analyzed. Details of the image processing scheme and its application will be presented.

\section{References:}

[1] R. Sharma, J. Mater. Res. 20 (2005) 1695.

[2] Sharma and Crozier in F Author in “Transmission Electron microscopy for nanotechnology”, ed.

Nan Yao \& Z.L Wang, (Springer Verlag and Tsinghua University press) p.531.

[3] https://imagej.nih.gov/ij/

[4] Yoshida et al, Nano Lett. 8 (2008) 2082

[5] Picher et al. Nano Lett. 14 (2014) 6104.
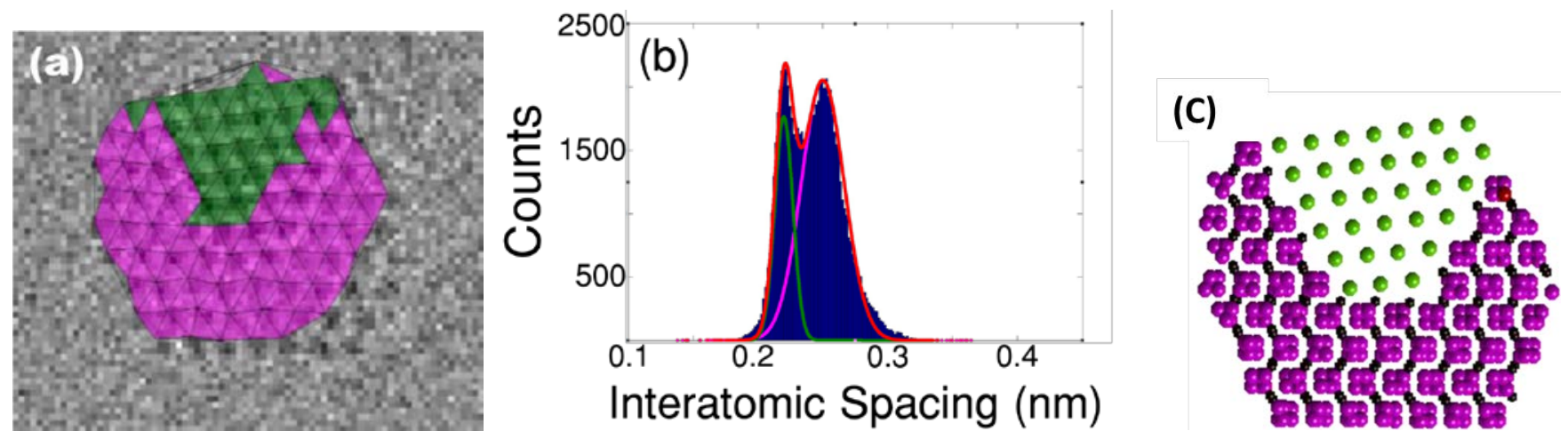

Figure 1. (a) Filtered high resolution image extracted from a video showing the existence of two phases, green and pink in a nanoparticle. (b) measured nearest neighbor distances are used to identify the green and pink regions as Co metal and Cobalt carbide structures, respectively. (a) Atomic model showing the relationship between the structures that was used for image simulation (not shown here).
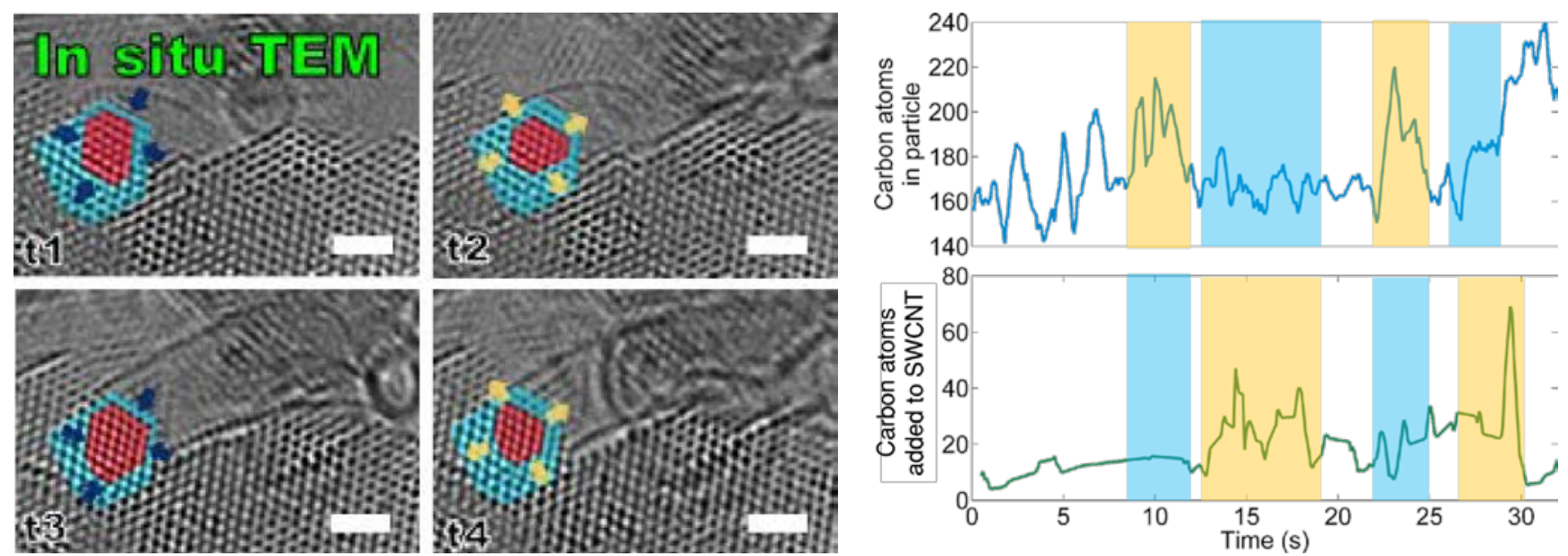

Figure 2. Sequence of 'unprocessed' time resolved images (left) showing the fluctuation in the area occupied by $\mathrm{Co}$ and $\mathrm{CoC}_{2}$ structures with time. The measured areas were used to calculate the fluctuation in the number of carbon atoms with time in the particle (top right) and compared with number of carbon atoms added to SWCNT during growth (bottom right). Areas shaded in blue and yellow in the graph show the increase in carbon content in carbide and SWCNT, respectively. 